\title{
Is dexmedetomidine really superior to propofol?
}

\author{
Sujoy Banik $^{1} \cdot$ Hemanshu Prabhakar ${ }^{1}$
}

Received: 4 March 2015 / Accepted: 10 March 2015 / Published online: 27 March 2015

(C) Japanese Society of Anesthesiologists 2015

\section{To the Editor:}

We read the article by Sriganesh et al. published ahead of print in your journal [1]. Based on a study conducted in 60 patients, the authors concluded that "dexmedetomidine appears to be superior to propofol as a sole sedative agent for sedation during cerebral angiography in patients with subarachnoid hemorrhage." We noticed that the sample size calculation by Sriganesh et al. is based on the onset time for sedation in pediatric patients undergoing magnetic resonance imaging. The validity of this extrapolation to cerebral angiography (CA) needs to be confirmed. We feel that the authors should have conducted a pilot study and then calculated the sample size. In an ongoing trial in our center, on a similar patient population with similar intervention drugs, we conducted a pilot study. We calculated the necessary sample size to be as large as 92 patients in each group in order to show significant differences in movement during CA. Sedation for diagnostic CA needs to be evaluated further in patients of different grades of subarachnoid hemorrhage, as the sensorium of the patients will significantly influence the choice of drug and its dosages.
Hemanshu Prabhakar

prabhakaraiims@yahoo.co.in

1 Department of Neuroanaesthesiology, Neurosciences Center, All India Institute of Medical Sciences (AIIMS), New Delhi 110029, India

\section{Reference}

1. Sriganesh K, Reddy M, Jena S, Mittal M, Umamaheswara Rao GS. A comparative study of dexmedetomidine and propofol as sole sedative agents for patients with aneurysmal subarachnoid hemorrhage undergoing diagnostic cerebral angiography. J Anesth. 2014. doi:10.1007/s00540-014-1952-1. 